\title{
Cyber-physical Systems - Von jeder mit jedem zu alles mit allem
}

\author{
Stefan Reinheimer $\cdot$ Susanne Strahringer
}

Eingegangen: 17. Oktober 2014 / Angenommen: 18. Oktober 2014

(C) Springer Fachmedien Wiesbaden 2014

Cyber-physical Systems sind die Grundlage für die so genannte vierte Industrielle Revolution - Industrie 4.0. Dieser Trend zur intelligenten Fabrik ist die Folge des Paradigmenwechsels von einfachen, autark agierenden Geräten, hin zu vernetzten, selbstständig miteinander kommunizierenden Systemelementen. In der Jubiläumsausgabe der HMD ist uns dies eine genauere Betrachtung wert.

Das Schlagwort und der Hype „Internet der Dinge“ bezeichnet Systeme, in denen die Kommunikation zwischen ganz normalen Alltagsgegenständen und Internetapplikationen stattfindet. So kann z. B. die Standheizung eines Autos mit einer App auf dem Smartphone über das Internet eingeschaltet werden. Aus unserer Vorstellung, dass das Web, insbesondere das Web 2.0, die Kommunikation und Vernetzung von Menschen untereinander verstärkt hat, wird durch das Internet der Dinge eine Realität geschaffen, in der alles mit allem vernetzt ist, egal ob Ding oder Mensch. Nachdem für die Kommunikation aber nicht ausschließlich Internetprotokolle Verwendung finden müssen, kam schnell die technisch neutralere Bezeichnung „Cyber Physical Systems“ auf. Der Begriff „Cyber“ ist dabei abgeleitet aus der Kybernetik, der Lehre von Regel- und Steuervorgängen, und tatsächlich sind in diesen Systemen Regelungs- und Steuerungsmechanismen von großer Bedeutung. Gleichzeitig wird „Cyber“ auch häufig als Präfix verwendet, um eine künstliche Realität anzudeuten. Demnach sorgen in cyber-physischen Systemen Sensoren und Aktoren in technischen Geräten für eine Verschmelzung der physischen Welt mit der virtuellen.

S. Reinheimer $(\bowtie)$

$\mathrm{BIK} \mathrm{GmbH}$,

90489 Nürnberg, Deutschland

E-Mail: stefan.reinheimer@bik.biz

S. Strahringer

TU Dresden, 01062 Dresden, Deutschland

E-Mail: susanne.strahringer@tu-dresden.de 
Dabei entstehen aus herkömmlichen Alltagsgegenständen cyber-physische Systemelemente. Sie erlauben eine hochgradig kontextspezifische Steuerung und neuartige, oft dezentrale Regelungsmechanismen.

Früher war ein Telefon ein Gerät zur Übertragung von Stimmen. Heute sind Smartphones Computer, mit denen man auch telefonieren kann, die aber durch ihre Verknüpfung mit dem Internet und Lokalisierbarkeit via GPS Systemelemente sind, die in eine Vielzahl von Prozessen eingebunden werden können. Sie lassen Ihre Freunde verfolgen, wo Sie sich gerade befinden und wie schnell Sie sich bewegen. Wenn Sie im Flughafen an einem Cafe vorbeikommen, erhalten Sie unvermittelt einen Gutschein für einen Espresso auf Ihr Handy. Dies ist Alltag.

Werden Autos, die ursprünglich als Ersatz für Pferdekutschen zu Transportzwecken entwickelt wurden, zukünftig nicht nur Informationen über die Verkehrsdichte austauschen, sondern evtl. sogar verhandeln, wer an der nächsten Kreuzung Vorfahrt hat? Können extern koordinierende Systeme wie Ampeln durch sich autonom abstimmende Smart Cars ersetzt werden, um den Verkehr kontextsensitiv und systemintern zu koordinieren?

Diese und andere Entwicklungen sind Ausdruck des Paradigmenwechsels von Alltagsgegenständen zu cyber-physischen Systemen. Die völlige Vernetzung vieler Gegenstände des täglichen Lebens ist nicht nur eine technologische Innovation, sondern sie verändert die Entwicklung neuer Produkte, die Optimierung von Prozessen, das soziale Zusammenleben, die Datensicherheit und den Alltag aller Menschen in der technologisierten Welt.

Die beiden Beiträge, die sich mit dem Thema „Cyber Physical Systems“ befassen, adressieren zwei der in diesem Umfeld für Unternehmen essenziellen Themenfelder. Gerade aus der Perspektive der Wirtschaftsinformatik von herausragender Bedeutung ist die Frage nach der Veränderung von Geschäftsmodellen in einer Welt, in der die Trennung von physischen und digitalen Branchen endgültig vorbei ist. Dieser Betrachtung ist daher der erste Artikel gewidmet. Ein zweiter Beitrag zeigt auf, dass sich Unternehmen, die CPS produzieren wollen, langfristig und grundlegend auf diesen Paradigmenwechsel vorbereiten müssen. Dabei sind sowohl ein fundamentaler organisatorischer als auch ein entwicklungsmethodischer Wandel zu bewältigen. Wir hoffen, dass Ihnen die Lektüre dieser Beiträge Hilfestellung gibt, die Konsequenzen dieses Paradigmenwechsels für Ihr eigenes Unternehmen einzuschätzen. 